(c)

(i) ${ }_{\text {ву }}$

$\ominus$ ND

() $\mathrm{Nc}$

\title{
EL PROCESO CREATIVO EN EL TAOÍSMO Y EN LA PSICOLOGÍA OCCIDENTAL
}

\author{
The Creative Process in Taoism and Western Psychology \\ O processo criativo no taoísmo e na psicologia ocidental
}

\section{RESUMEN}

Mostrando algunas analogías entre el pensamiento oriental, especialmente del antiguo taoísmo chino, y algunas escuelas psicológicas contemporáneas cercanas al neohumanismo gestáltico y la llamada psicología transpersonal, en el presente artículo se presentan y discuten los principales hallazgos de la psicología sobre el proceso creativo, las características de personalidad de los sujetos altamente creativos y las circunstancias psicosociales que suelen favorecer el surgimiento de la creatividad. Traza una línea en el espacio y ya tienes un lado y el otro. Piensa en algo y te diré que lo contrario también existe con su monto de verdad. Justifícame el ser, que yo te justificaré el no ser. Arriba y abajo, izquierda y derecha, vida y muerte, belleza y fealdad, bien y mal, éxito y fracaso, luz y oscuridad, tristeza y alegría, etc., los anteriores son apenas algunos ejemplos de conceptos contrarios que, tal vez, son realmente opuestos en la realidad o solo son concebidos como tales por la forma en la cual funcionan nuestros procesos sensoriales y cognitivos.

EVALUADO:31DEJULIO DE 2013

Hugo Mastrodoménico Brid (Colombia)

Magíster en Mercadeo

Universidad Jorge Tadeo Lozano

hugo.mastrodomenico@utadeo.edu.co

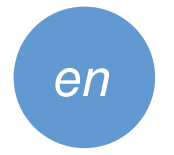

\section{ABSTRACT}

Through some analogies between eastern thinking, especially that of the ancient Chinese Taoism, and some contemporary schools of psychology related to the gestaltic neo-humanism and the so called trans-personal psychology, this paper presents and discusses the main findings of psychology on the creative process, the personality traits of highly creative subjects and the psychosocial circumstances that usually favor the appearance of creativity. Draw a line in space and now you have one side and the other. Think about something and I will tell you that the opposite also exists with its measure of truth. Justify "being" to me, and I will justify "not being". Up and down, left and right, life and death, beauty and ugliness, good and evil, success and failure, light and darkness, sadness and happiness, etc. Those are just some examples of opposite concepts that, maybe, are truly opposite in reality or are just conceived as such due to the way in which our sensory and cognitive processes work.

\section{RESUMO}

Mostrando algumas analogias entre o pensamento oriental, especialmente do antigo taoísmo chino, e algumas escolas psicológicas contemporâneas próximas ao neohumanismo gestáltico e a chamada psicologia trans-pessoal, no presente artigo se apresentam e discutem as principais descobertas da ciência psicológica sobre o processo criativo, as características de personalidade dos sujeitos altamente criativos e as circunstâncias psicossociais que costumam favorecer o surgimento da criatividade. Traça uma linha no espaço e já tens um lado e o outro. Pensa em algo e te direi que o contrário também existe com seu monto de verdade. Justifica-me o ser, que eu te justificarei o não ser. Arriba e abaixo, esquerda e direita, vida e morte, beleza e fealdade, bem e mal, sucesso e fracasso, luz e obscuridade, tristeza e alegria, etc., os anteriores são apenas alguns exemplos de conceitos contrários que, tal vez, são realmente opostos na realidade ou só são concebidos como tais pela forma na qual funcionam nossos processos sensoriais e cognitivos.

\section{PARA CITAR ESTE ARTÍCULO/TO CITE THIS ARTICLE/PARA CITAR ESTE ARTIGO:}


El pensamiento oriental siempre ha resultado exótico entre nosotros. Los orientales desarrollaron desde mucho tiempo atrás una forma alterna de concebir la realidad y relacionarse con ella. No obstante, en el seno de la psicología han logrado acogida en los últimos años algunas corrientes teóricas que han buscado acercarse al pensamiento oriental y han hecho palpables ciertas coincidencias entre ambas concepciones, como ocurre en la teoría de la Gestalt ${ }^{1}$ o de la llamada psicología transpersonal (González, 1992).

Tanto en Oriente como en Occidente el concepto de creación incluye una dimensión espiritual, puesto que la creación del universo está intrínsecamente asociada al concepto de Dios. En el taoísmo, dicho concepto se encuentra planteado poéticamente en el Tao Te Ching o El libro del camino y la virtud (Yutang, 1953).

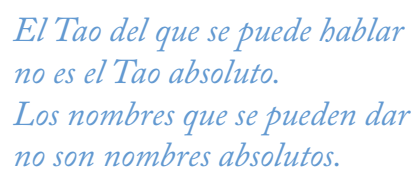

Lo innombrado es el origen del universo.

Lo nombrado es la madre de todas las cosas.

El aparente juego de palabras con el que empieza el Tao Te Ching, atribuido a Lao-tse (siglo VI a. C.), está expresando una profunda verdad psicológica y trascendental que nos ayuda a comprender la forma en que el taoísmo concibe el papel de la creatividad en la experiencia humana. El taoísmo, al igual que otras importantes cosmovisiones orientales, no intenta personificar a Dios y plantea la existencia de un mundo del pensamiento y un mundo de los sentidos. Sin embargo, de una manera tangencial, los taoístas sí reconocen a la divinidad como principio desencadenante de todo lo existente; es más, si alguna vez lo describen con una denominación, lo hacen hablando de "lo creativo", en contraposición al otro principio que actúa como su opuesto: "lo receptivo".

El principio receptivo es, por su esencia, el principio femenino, el encargado de gestar y dar existencia a las cosas perceptibles en el mundo material, el mundo sensible. Pero para que las cosas puedan tener existencia material, primero deben haber sido concebidas en el mundo de las ideas. El tao que se manifiesta en el mundo, puesto que ya está diferenciado en términos particulares, puede ser designado con denominaciones que señalan sus límites respecto de las demás particularidades y respecto del todo universal.

Dentro de tal concepción, que en Occidente calificaríamos como idealista, hasta se podría llegar a pensar que es la denominación misma la que le da existencia a las cosas. Esta línea de ideas se soporta en que, aunque la realidad existe independientemente de la percepción humana, el hombre solo puede concebir la existencia de las cosas materiales con su experiencia sensorial y su subjetividad que incluye el entendimiento interpretativo de todo lo existente mediante una denominación simbólica de cada elemento, denominación e interpretación que es compartida, validada y retransmitida por un colectivo de personas. Con todo, desde la perspectiva de un sujeto, la existencia de las cosas es una existencia "para sí", más que una existencia "en sî".

Pero el argumento inicial del Tao Te Ching radica en que cuando se intenta limitar mediante las palabras el principio creador en su sentido universal, se hace evidente que el lenguaje y el pensamiento categorizador no pueden abarcar lo que por su misma naturaleza es inefable, dado que está más allá del ser o del no ser. Entonces, puesto que los nombres

\footnotetext{
1 Gestalt:palabra alemana que significa forma o estructura. Su traducción debe hacerse como 'configuración'. Se trata de una escuela psicológica que realizó investigaciones experimentales en el campo de la percepción las cuales culminaron en la formulación de las llamadas leyes de la Gestalt que explican el proceso por el cual captamos la realidad. Uno de sus postulados más significativos hace hincapié en "el todo es más que la suma de sus partes", con lo que se expresa que los sistemas que se configuran a partir de la integración de varios subsistemas aparecen en la realidad con características propias que no estaban en los subsistemas iniciales, sino que son frutos de la integración misma. Asi, un cuerpo no solo es un corazón, un higado, etc., sino que se trata de un todo, una entidad que funciona autónomamente y tiene su propia dinámica inherente a su organización como tal.
}

I Panorama 
se refieren a lo particularizado e individualizado, ningún nombre puede designar con justicia lo que por su esencia es universal e inmanifiesto.

Ahora bien, una vez manifiestas todas las cosas, el ser humano solo puede interpretarlas en su relación de unidad y confrontación con su opuesto.

En la búsqueda de puntos de encuentro entre el taoísmo y la psicología occidental, algunos autores han encontrado seductor el concepto chino de lo creativo tal y como está desarrollado en el $I$ Ching, también conocido como El Libro de los caminos y las mutaciones. E1 I Ching es un antiguo texto milenario que ha sido empleado a lo largo de los siglos como oráculo y también como depositario de una profunda sabiduría que ayuda a los hombres en los asuntos mundanos y en los espirituales (figura 1).

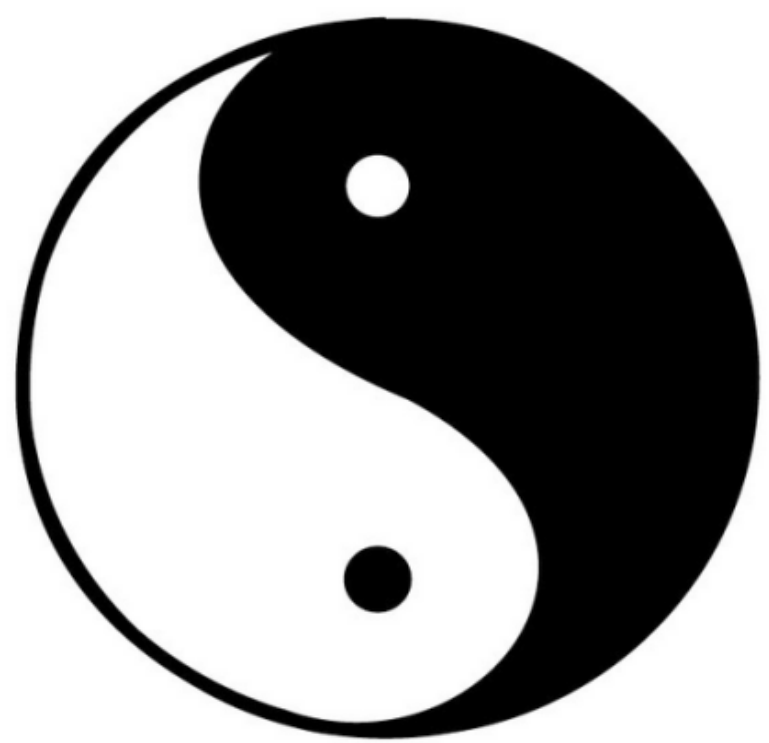

FIGURA 1.TAICHI, LA UNIDAD DEL YIN Y EL YANG.

Como se explicó, los chinos no personifican la divinidad, pues prefieren resaltar su interacción con la realidad humana en su manifestación mundana. Esto se expresa magníficamente en el famoso símbolo de taichi que conjuga el yin y el yang. En este símbolo, los dos elementos contrarios se complementan y conjuntamente forman una unidad que los incluye, pero, además, encontramos un punto claro dentro del elemento oscuro y un punto oscuro dentro del claro; dichos puntos están indicándonos que cada elemento posee en su seno el germen de su contraparte. Esto ilustra la fuerte creencia china de que los opuestos solo pueden existir en su interacción con su antagonista.

El elemento yang es lo masculino, lo positivo, el día, el sol, lo activo, la vida, etc. Yin incluye lo femenino, lo negativo, la noche, lo pasivo, la muerte. Con respecto a la mente, el yang es lo consciente en tanto que lo inconsciente es el yin (Dethlefsen y Dahlke, 1998) (figura 2).

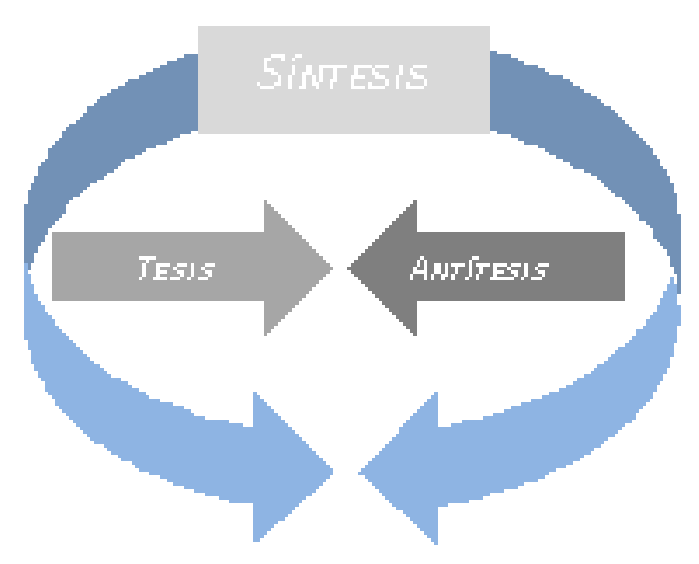

FIGURA 2. LA UNIDAD Y LA LUCHA DE LOS CONTRARIOS.

Esta concepción de la realidad basada en la unidad y la lucha de los contrarios que de entrada son entendidos como complementarios fue expuesta en Occidente por Hegel con su planteamiento dialéctico (Ferrater, 1971) y ha sido utilizada para explicar los modernos hallazgos de la física cuántica (Capra, 1987). Igualmente, no hay que olvidar que todos los desarrollos de la electrónica se basan en un modelo binario de unos y ceros. El yin y el yang no conforman un modelo dualista, se trata de una noción que en últimas es monista, pues al integrar los dos antagonistas en una unidad que los contiene surge un concepto que se encuentra en un nivel superior. Es la síntesis que incluye la tesis y la antítesis para luego entrar en una contradicción con una antítesis también superior. De esta manera, ambos aspectos de la contradicción inicial son imprescindibles y se requieren mutuamente. Pero para iniciar el movimiento es esencial que un aspecto rompa el equilibrio de la tensión y pase a predominar sobre el otro y se establezca una jerarquía. No obstante, tal predominio es solo temporal, y con el tiempo le llegará el momento de predominar al elemento opuesto. Así, por ejemplo, cuanto más asciende la luz del sol, más próxima se encuentra la llegada de la noche, que también prevalecerá por un tiempo, pero igualmente encontrará su declive. 
Esta concepción, que es eminentemente dinámica al asumir que el cambio es lo único que es permanente, presenta una gran analogía con una de las leyes de la Gestalt, la de la ley de figura-fondo que nos dice que al percibir la realidad nosotros siempre seleccionamos uno de los elementos presentes al que le damos prioridad y lo captamos en primer plano, con riqueza de detalles y a profundidad (la figura); al mismo tiempo, le restamos importancia a los demás elementos presentes a los que ubicamos en un segundo plano y los captamos difusos $\mathrm{y}$ sin detalles (el fondo). Nos es imposible captar simultáneamente dos elementos como figura, pero podemos cambiar rápidamente el elemento que ocupa el primer plano. Este proceso es la atención y no se aplica solo a lo visual, sino a todos los sentidos y ocurre entre todos ellos juntos, y en opinión de algunos teóricos, como Fritz Perls, rige todas las funciones psíquicas (Perls, 1987; Feldman, 1998). Siendo así, no podemos pensar en dos cosas al mismo tiempo ni procesar dos recuerdos simultáneamente; le damos prioridad a un elemento en tanto que los demás le proporcionan un marco o un escenario de fondo, ocupando un segundo plano. En la figura 3, por ejemplo, no es posible percibir al mismo tiempo los ángeles y los demonios, aunque se puede cambiar rápidamente entre unos y otros.

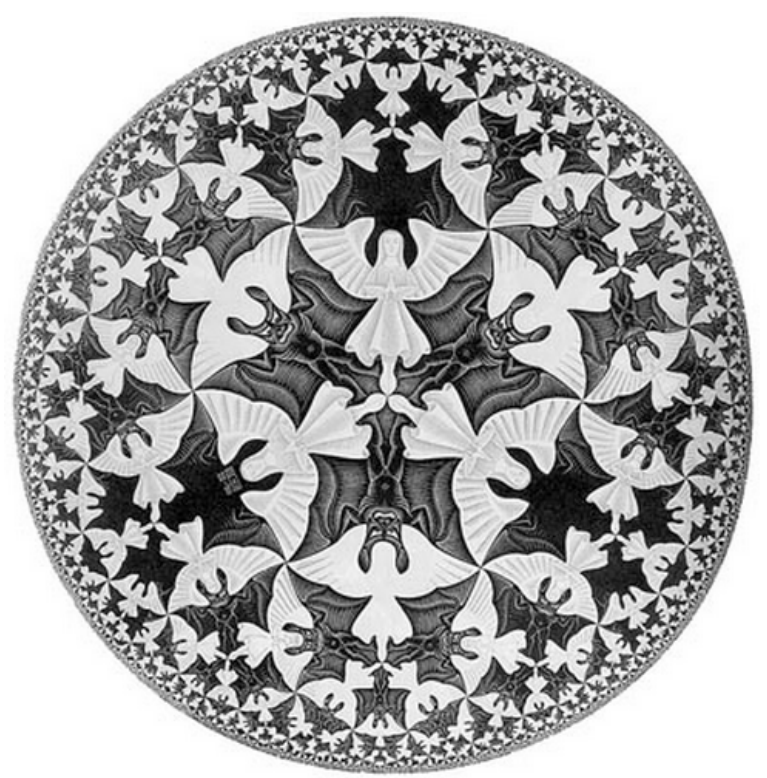

FIGURA 3. APLICACIÓN DE LA LEY DE FIGURAFONDO. OBRA DE M. C. ESCHER.

Otro principio gestáltico, la ley del cierre, también nos ayuda a comprender la creatividad. El cierre ocurre cada vez que le damos finalización a un ciclo o asunto inconcluso, lo que implica la disminución de la tensión inherente a él. Cuando cerramos un ciclo de inmediato deja de ser figura en nuestra mente y se desplaza hacia el fondo. El siguiente proceso, que se experimenta como “¿y ahora qué?, es la búsqueda de la tensión mayor que implica elegir un nuevo asunto inconcluso que requiere nuestra atención y nuestra dedicación para lograr un nuevo cierre. En este sentido, el acto creativo es un cierre que conlleva el descubrimiento de una relación no concebida antes entre los elementos que generaban la tensión. Los teóricos de la Gestalt denominaron insi$g h t$, comprensión súbita cercana a la intuición, a aquel momento cumbre en el que de manera aparentemente casual se logra una solución creativa a un problema. El cierre, por supuesto, es un todo que contiene algo más que la suma de sus partes.

Volviendo a la concepción china, en los trigramas y hexagramas del I Cbing, sus antiguos creadores encontraron una forma matemáticamente perfecta de representar la complejidad que resulta de combinar todas las posibles relaciones entre el primer par de elementos opuestos representados por una línea continua, el yang, y una línea partida, el yin. Al combinarse estas líneas nos dan cuatro posibilidades: el yang joven y el viejo, y el yin joven y el viejo. Al agregarse otra línea aparecen los ocho trigramas simbolizados por una familia en la que el padre, chien, está conformado por tres líneas continuas y la madre, kun, por tres líneas partidas, los tres hijos poseen dos líneas partidas y una continua, en tanto que lo opuesto ocurre con las tres hijas. Cuando cada uno de estos trigramas se combina con los demás, surgen los 64 hexagramas que, gracias a las relaciones entre ellos, representan todas las situaciones posibles (figura 4).

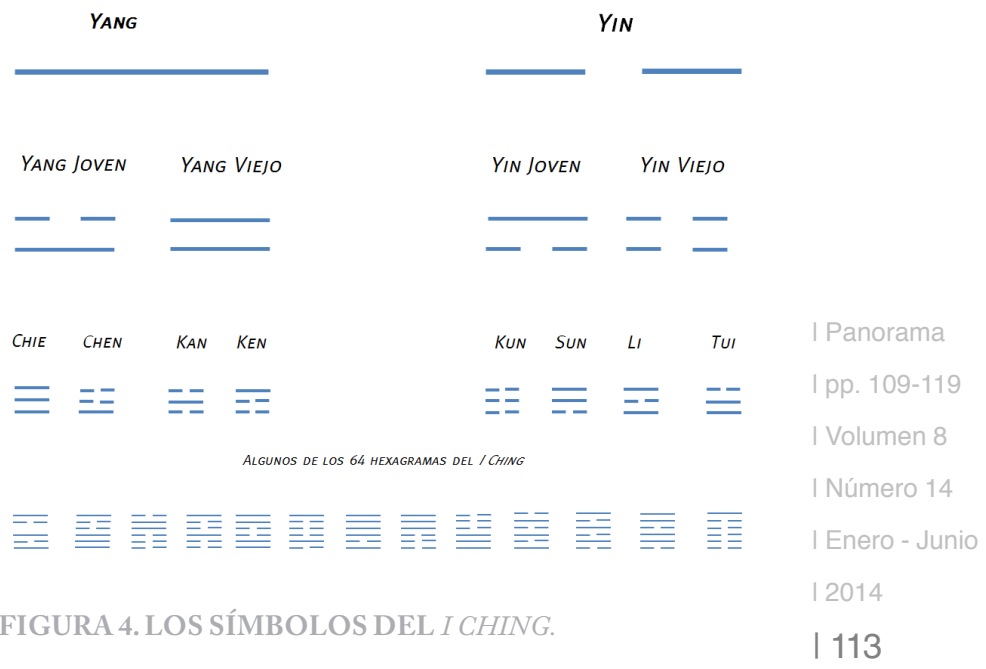




\section{LO CREATIVO Y LO RECEPTIVO}

El primer hexagrama del I Ching es lo creativo, es un signo que se compone de seis trazos continuos, representando la protoenergía o energía primaria, luminosa, fuerte, espiritual y activa. El signo es total y uniformemente fuerte en su naturaleza. Puesto que no lo afecta ninguna debilidad, es en sí mismo, de acuerdo con su naturaleza intrínseca, la fuerza, la energía. Su imagen es el cielo. Se trata de lo único que no está condicionado por las circunstancias. Es la poderosa acción creativa de la divinidad (Wilhelm, 1982).

El segundo hexagrama es lo receptivo, todas las líneas que componen el signo son partidas. Es lo derivado. La cualidad intrínseca del signo es la entrega ferviente, su imagen es la tierra, lo material. Lo receptivo logra su trascendencia dando manifestación corpórea y sensible a lo que antes permanecía en el mundo de las ideas, es la expresión del impulso espiritual de lo creativo. Es su contraparte y su complemento.

La oposición entre lo creativo y lo receptivo es solo aparente, ambos forman la unidad de la naturaleza y el espíritu, de lo espacial frente a lo temporal, de lo maternal frente a lo paternal, del cuerpo frente a la mente, etc. Se trata de lo que es (figura 5).

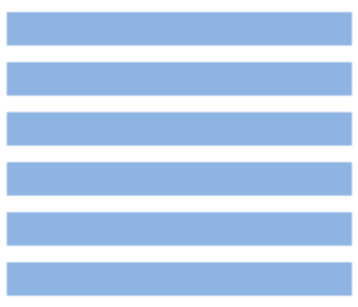

Io Cieatsvo

CONCEP OEN OE LA JOEA

Materializaobn oe La joea pp. 109-119 |

Volumen 8 ।

Número 14 I

Enero - Junio I

2014 ।

\section{EL PENSAMIENTO CREATIVO PARA LA PSICOLOGÍA OCCIDENTAL}

Tal vez, no sea casualidad que el cerebro humano esté conformado por dos hemisferios que poseen funciones 114 |

FIGURA 5. LOS DOS PRIMEROS HEXAGRAMAS DEL I CHING. diferentes que terminan complementándose. Así, el he- misferio izquierdo procesa la lógica de los argumentos, se ocupa de los aspectos gramaticales y sintácticos del lenguaje, de la lectura y la escritura, del cálculo, de los procesos analíticos, de la atribución de causalidades y de la noción del tiempo. A su vez, el hemisferio derecho se encarga de la percepción de las formas, de la comprensión de la música, de lo gráfico, de encontrar las analogías y, en fin, de los procesos de síntesis y de comprensión general (Dethlefsen y Dahlke, 1998).

Dada la enorme variedad de teorías psicológicas existentes, la concepción gestáltica dista mucho de ser la única que nos da una explicación acerca de la creatividad. Prácticamente, todas las escuelas psicológicas occidentales nos presentan una concepción más o menos diferente del acto creativo. Los primeros intentos los encontramos en el asociacionismo que explica todos los procesos del pensamiento como conexión de ideas derivadas de la experiencia. En este sentido, el pensamiento creativo representaría una conexión entre ideas más bien alejadas entre sí como fruto de un proceso imaginativo casual. El conductismo radical, por su parte, nos diría que una persona aprende a ser creativa por su interés en obtener recompensas y evitar castigos y luego simplemente pone en ejecución las conductas que antes le dieron resultados favorables. En tal sentido, el énfasis está puesto en el ambiente (González P., 2006).

Las teorías psicodinámicas, más o menos cercanas al pensamiento original de Freud, entienden la idea creativa como una solución de compromiso entre las fuerzas que se encuentran en conflicto en la psiquis de cada individuo. Así, las pulsiones sexuales culpabilizantes son sublimadas, es decir, caracterizadas conscientemente como encaminadas a lograr un fin noble, pero en el fondo esconden motivos egoístas y hedonistas. En Adler, se trataría de compensar un sentimiento de inferioridad y en el de Jung se trataría de conciliar disposiciones opuestas.

Por otra parte, la teoría humanista de Maslow y Rogers, con su visión optimista de la naturaleza humana, presupone que en el hombre existen tendencias a construir un ser personal superior al que somos, un individuo autorrealizado que ejercita su ser creativo como expresión de su crecimiento personal y su integración al contexto sociocultural. Ideas similares son planteadas por la teoría de las inteligencias múltiples de Gardner (González H., 2012). Otros autores, como Mihaly Csikszentmihalyi 
(2008), también plantean ideas cercanas al privilegiar estados mentales específicos que propiciarían el surgimiento de las ideas creativas. Tal es el llamado estado de flujo que ocurre cuando la persona alcanza cierto nivel de experticia en un campo que también se ve acompañado de un grado de exigencia situacional que, sin llegar a constituirse en una presión desmedida, sí alcanza a estimular a la persona.

Curiosamente, Jean Piaget, uno de los grandes de la psicología especialmente en el campo de los procesos cognitivos, con su inusual enfoque de combinar su formación de biólogo con su intento de resolver problemas filosóficos en lo que denominó como estructuralismo genético, terminó planteando una concepción de la creatividad que también presenta grandes analogías con el pensamiento chino. Para Piaget, la vida es una creación continua de formas que tienen lugar por el proceso dialéctico entre asimilación y acomodación ${ }^{2}$. Esta interacción le permite al individuo construir estructuras cognoscitivas cada vez más complejas que hacen que la persona no sea receptora pasiva de las influencias del ambiente, sino que actúa sobre ellas modificando el mundo en el que vive (Del Val, 1980).

Si intentamos mencionar los principales hallazgos sobre la creatividad desde una perspectiva psicológica menos teórica y más práctica, encontramos que algunos autores hacen hincapié en el aspecto de combinar respuestas o ideas de manera novedosa (Feldman, 1998). Otros autores se centran en que los productos del proceso creativo sean socialmente útiles (Morris y Maisto, 2001), en tanto que otros agregan la importancia de que sean significativos (Garrison y Loredo, 2002) (figura 6).

\footnotetext{
2 De nuevo nos encontramos con otro par de elementos contrarios y complementarios. En la asimilación, lo que se está conociendo solo puede ser interpretado a partir de lo ya conocido, en tanto que en la acomodación lo ya conocido se reconfigura por cuenta de lo que se acaba de conocer.
}

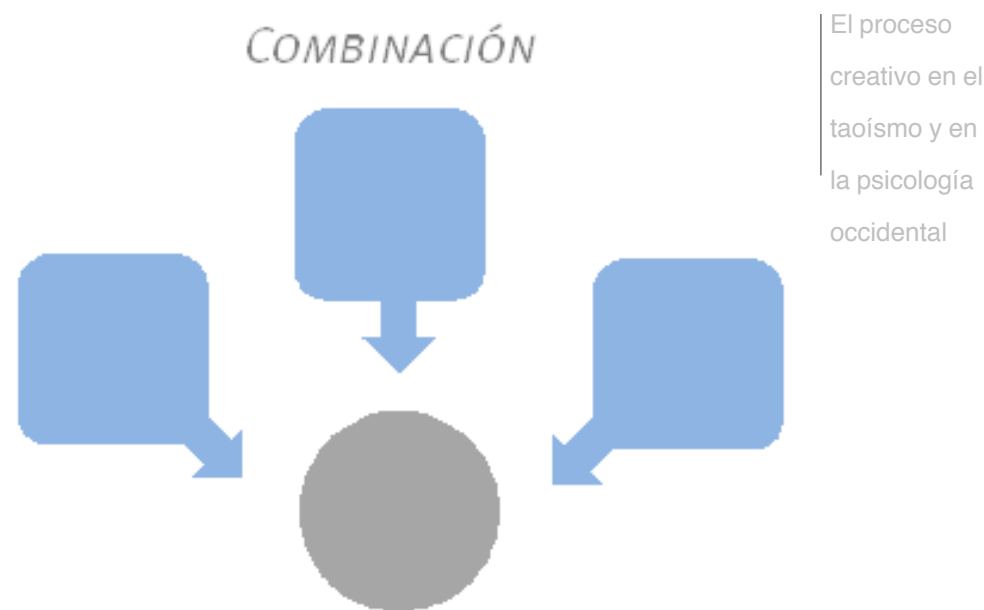

FIGURA 6. EL ACTO CREATIVO RESULTADO DE HALLAR NUEVAS RELACIONES ENTRE LO YA CONOCIDO.

Se acepta que un creador no desarrolla su producto partiendo de la nada, su aporte consiste en establecer una relación antes no concebida entre elementos ya existentes. Así, la rueda fue inventada hace miles de años; las maletas por su parte existen hace, tal vez, cientos de años. Durante mucho tiempo las maletas fueron cargadas o arrastradas con mucho esfuerzo por sus dueños. Se necesitó un creativo que hiciera el nexo y se le ocurriera la idea de ponerles ruedas. La combinación no solo resultó novedosa, sino útil y significativa por cuanto las maletas con ruedas son objetos que hoy hacen parte de la vida cotidiana de millones de personas en el mundo. El anterior ejemplo nos ilustra cómo la acción de crear pasa por concebir una combinación original de elementos que antes se veían inconexos y cómo el producto final ha de cumplir una función utilitaria y socialmente significativa. Volviendo al planteamiento gestáltico, no sobra reiterar que una maleta con ruedas es un todo organizado que termina superando a sus componentes separados (figuras 7 y 8 ).

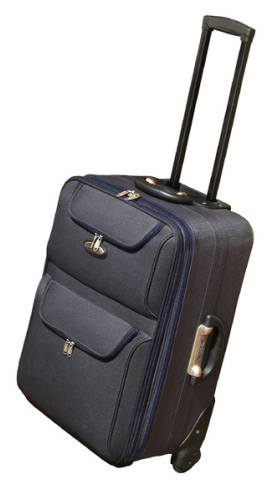

I Panorama I pp. 109-119 I Volumen 8 I Número 14 I Enero - Junio FIGURA 7. EL TOD O ES MÁS QUE LA SUMA DE SUS | 2014 PARTES. 


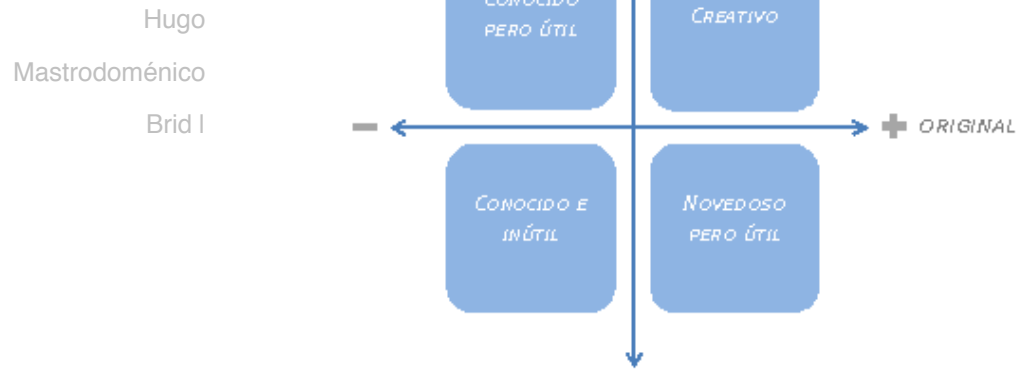

FIGURA 8. LO CREATIVO NO SOLO DEBE SER NOVEDOSO SINO ÚTIL.

En otros casos, el producto creativo consiste en "descubrir" un proceso natural. El avance estriba en que las personas logran comprender lo que antes no entendían y al hacerlo logran darle aplicaciones prácticas. Tal cosa ocurrió con el perfil aerodinámico: las aves siempre han maravillado a los hombres con su vuelo y nunca faltaron los intentos de imitarlo. Fue necesario que alguien comprendiera un principio físico simple: las partículas de aire se demoran más en pasar por una superficie curvada que por una plana, la diferencia genera un fuerza de sustentación que permite el vuelo si un cuerpo con tal perfil se desplaza en el aire a una velocidad mínima (figura 9).

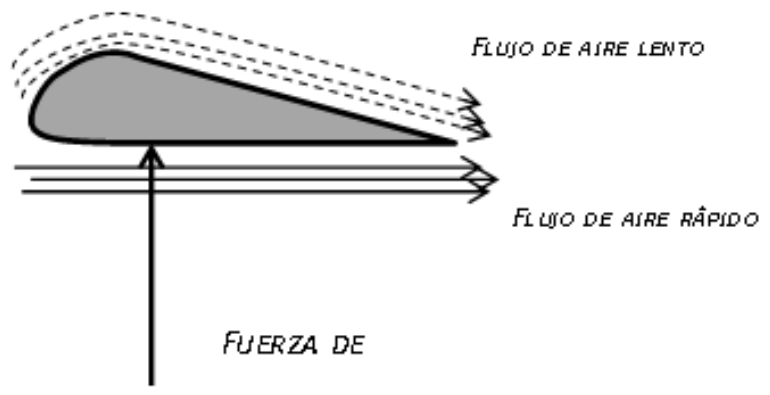

FIGURA 9. EL PERFIL AERODINÁMICO.

Panorama I pp. 109-119 |

Volumen 8 ।

Número 14 |

\section{LA PERSONA CREATIVA}

Thomas Alva Edison, una de las personas más reconocidas por su gran talento creativo, expresó que su obra tiene $1 \%$ de inspiración y $99 \%$ de transpiración. Pablo Picasso, por su parte, afirmaba: "Cuando llegue la inspiración, que me encuentre trabajando”. Inspiración y esfuerzo son dos cosas que conforman también un dúo de contrarios y complementarios, pero su contrajuego que materializarla. Aunque es imposible medir la magnitud de la participación de cada una de las dos partes, es obvio que el papel de la inspiración es mucho mayor de $1 \%$ y en muchos casos es el aspecto predominante, y el decisivo.

La inspiración ha sido observada en los primates superiores, como en algunos chimpancés que parecen experimentar un momento de revelación (insigtb) y repentinamente logran encontrar la solución de un problema (Feldman, 1998). Se trata de un aprendizaje que no tiene lugar por ensayo y error ni por observación e imitación, surge como un descubrimiento súbito. Es importante aclarar que no todos los chimpancés logran encontrar tal solución y que este tipo de procesos son cada vez más escasos en la medida en que descendemos en la escala evolutiva.

Si bien todos los seres humanos tenemos desarrollada la facultad creativa, no es cierto que todas las personas sean altamente creativas. Es evidente que muchas de las grandes creaciones se deben a personas especiales. Una característica notoria en los creativos es el predominio en ellos del pensamiento divergente sobre el convergente (otra dualidad). El pensamiento convergente se fundamenta en los conocimientos y en la lógica y suele producir respuestas previsibles y similares entre la mayoría de las personas (figura 10).

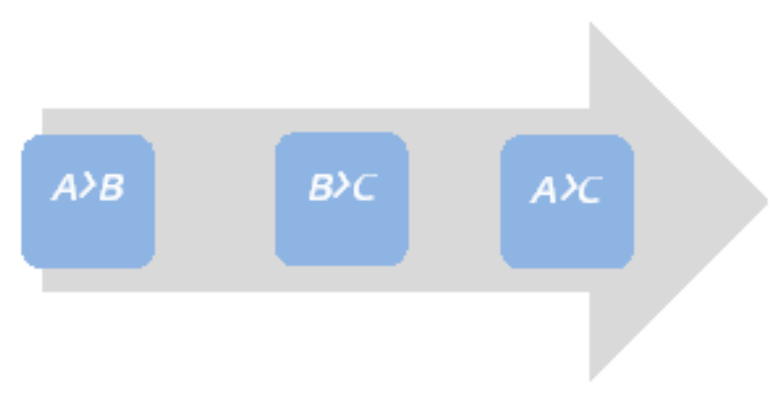

FIGURA 10. PENSAMIENTO CONVERGENTE.

Pero algunos individuos presentan una tendencia a salirse de los esquemas que les son planteados y buscan una salida alterna que de todas maneras pueda aportar soluciones ajustadas a los requerimientos de la situación. 


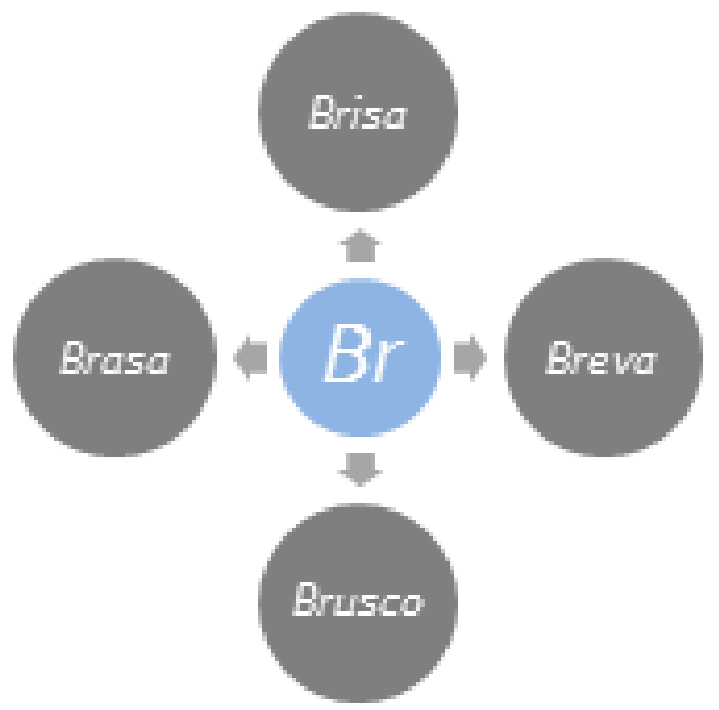

FIGURA 11. PENSAMIENTO DIVERGENTE.

En un plano emocional, el empleo del pensamiento divergente implica también una mayor disposición a correr riesgos y a hacer cosas diferentes de las que hacen la mayoría de las personas. Esta disposición es un rasgo de la personalidad que tiene mucho que ver con aspectos situacionales, pero es bueno tener presente que un gran número de estudios han encontrado que este rasgo está asociado a otro: la confianza en sí mismo (Schiffman y Kanuk, 2001).

Otra característica que suele acompañar a las personas creativas es su complejidad cognitiva. En este caso se trata de un rasgo que los lleva a internarse en la exploración y evaluación de ideas abstractas, elaboradas, intrincadas y llenas de matices. Igualmente, los creativos suelen tener multiplicidad de intereses y conocimientos, esto los lleva a poseer un arsenal mayor de elementos a la hora de efectuar las combinaciones requeridas para hallar las ideas originales (Feldman, 1998).

Aparte de lo ya mencionado, una lista más completa de las características que comúnmente suelen poseer los individuos altamente creativos incluye desarrollo de la facultad crítica, independencia de criterio, curiosidad, empleo frecuente de analogías, buena capacidad imaginativa, búsqueda frecuente de soluciones alternas, disciplina en el trabajo, tolerancia a la ambigüedad e intuición.

Pero es bien sabido que los rasgos de personalidad no son entes autónomos, se encuentran predispuestos en la configuración genética que recibe el individuo de sus progenitores y también se hallan favorecidos o refrenados por las circunstancias sociales y culturales en las que se desenvuelve el sujeto. En este sentido, las experiencias familiares y las vivencias de la primera infancia han demostrado ser muy importantes.

Sobre este asunto, como en todos dirían los chinos, hay dos posturas encontradas. Por una parte, la llamada teoría del gran hombre que hace hincapié en la posesión por nacimiento de ciertas características geniales que actuarían como la causa fundamental de la creatividad. Del lado opuesto está la teoría situacional que pone en primer plano el aprendizaje que a veces se obtiene gracias a un ambiente que favorece el desarrollo de un potencial presente en todas las personas. Como siempre, las dos teorías tienen algo de razón, y en parte están equivocadas. La equivocación de ambas consiste en negar la importante participación del aspecto contrario. Es un error considerar a todas las personas como poseedoras de iguales potencialidades. Si algo ha quedado claro en los últimos años de investigación en genética es que hay características cognitivas y emocionales altamente asociadas a la presencia o ausencia de ciertos genes (Meyer, 1996). Pero el biologismo radical también se equivoca al desconocer la importancia del condicionamiento social y cultural de las características psicológicas incluso el pensamiento altamente creativo. Se ha demostrado que las experiencias vividas en los primeros años afectan directamente la forma en que se conectan los circuitos neuronales en el cerebro con lo que su operatividad fisiológica queda condicionada a ser poco o muy sensible a ciertas circunstancias; es como si el cerebro se preparara adaptativamente para un tipo específico de vida partiendo de lo que la persona encuentra en las situaciones que experimenta en los primeros sucesos que enfrenta (Teicher, 2002). Pero, además, es evidente que la sociedad y la cultura aportan el sentido de las experiencias mentales.

No debe entonces perderse de vista que los seres humanos somos unidades bio-psico-sociales, es decir, somos organismos sujetos a las leyes biológicas; también somos seres sociales, inmersos en los patrones culturales que prevalecen en nuestro entorno específico que nos condicionan en un sentido u otro; y también somos seres conscientes, poseedores de una mentalidad que nos hace únicos y que a veces nos permite concebir al mundo de una manera diferente para poder transformarlo.
El proceso creativo en el taoísmo y en la psicología occidental
I Panorama I pp. 109-119 I Volumen 8 I Número 14 I Enero - Junio | 2014 


\section{CONDICIONANTES SOCIOCULTURALES DE LA CREATIVIDAD}

Al considerar algunas circunstancias situacionales que estarían contribuyendo al desarrollo de una personalidad creativa, las opiniones están divididas en cuanto a si las privaciones y carencias ayudan al desarrollo de la inventiva o si, por el contrario, la posesión de las mejores condiciones y todos los insumos para lograr ese desarrollo es el escenario más fructífero. En nuestro medio es común escuchar comentarios que apoyan la primera opción. Se afirma que las grandes carencias nos obligan a esforzarnos por encontrar salidas alternativas; no obstante, es obvio que para lograr muchos desarrollos es indispensable la tenencia de condiciones mínimas que hagan posible el encuentro de la nueva relación.

Algunos autores han aventurado una hipótesis que ha encontrado cierto apoyo en la investigación aunque, dada la gran cantidad de variables que intervienen en la configuración de la personalidad humana, no es posible hacer afirmaciones generales ni absolutas al respecto. Se trata del orden de nacimiento. La evidencia indica que los hermanos mayores tienden al pensamiento convergente en tanto que los menores al divergente. Esto estaría dado por la dinámica familiar en la que los hermanos se disputan los diferentes nichos afectivos disponibles en el seno de la familia. En efecto, el hermano mayor, digamos de unos 4 años, es inmensamente superior al menor que tendría alrededor de 2 años. Es superior en tamaño, fuerza, capacidad de expresión, comprensión de la complejidad de las situaciones y en muchas otras cosas. El hermano mayor tiene desde el comienzo toda la atención y el apoyo de los padres y otros familiares. $\mathrm{Su}$ estrategia para conservar esa condición es "portarse bien”, es decir, seguir el camino señalado, hacer y decir lo que se espera de él y hacerse defensor del orden establecido y de la autoridad. Sus ideas tienden a ser conservadoras y sus estrategias de pensamiento a ser convencionales. Esto le permite ir directamente hacia las recompensas afectivas que proporciona la familia. Para el hermano menor la misma estrategia no es $\tan$ rentable; el menor siempre estará en segundo lugar, pues el primogénito lo supera ampliamente en todos los terrenos. Por ello, la situación lo obliga a buscar "la otra forma de", el camino alternativo. En la medida en que sus capacidades le permitan encontrar soluciones diferentes de los problemas cotidianos, la exploración de lo diverso puede ser la forma de obtener la atención y el reconocimiento necesario.

Hay estudios que muestran que muchos de los grandes innovadores en la historia de la humanidad han sido hermanos menores. Pero se trata de una dinámica muy compleja en la que también intervienen otras variables, como el sexo de cada uno de los hermanos, las diferencias de edad, el número total de hermanos, la inteligencia de los padres, su nivel educativo, la ideología que profesan, los rasgos de personalidad que poseen y el trato específico que le dan a cada uno de sus hijos. A pesar de tal complejidad, la mayor predisposición de los hermanos menores a seguir el camino alterno puede establecerse empíricamente (Sulloway, 1997).

La búsqueda de ese camino alterno, de esa "otra forma de", es uno de los grandes motores de la civilización. Debido a que nunca faltan las personas que no se conforman con los esquemas imperantes y se esfuerzan por encontrar otros paradigmas, surgen nuevos avances que la sociedad acoge, acumula y retransmite a las siguientes generaciones. Por duro que resulte decirlo, los líderes políticos y religiosos han propiciado pocos cambios significativos en la cotidianidad de la vida de las personas, los innovadores y los creativos son los responsables de la inmensa mayoría de tales cambios. Por ello, el pensamiento chino siempre ha privilegiado la contradicción y prefiere aceptarla como tal en vez de temerle.

\section{REFERENCIAS BIBLIOGRÁFICAS}

1. Baron, R. y Byrne, D. (1998). Psicología social. Madrid: Prentice Hall Iberia.

2. Capra, F. (1987). El tao de la física. Una exploración de los paralelos de la física moderna y el misticismo oriental. Madrid: Luis Cárcamo.

3. Csikszentmihalyi, M. (2008). Fluir. Una psicologia de la felicidad. Barcelona: Kayros.

4. Val, J. del (1980,18 de septiembre). Jean Piaget, la obsesión por la creatividad. El País, España. Recuperado de 2013 http://elpais.com/diario/1980/09/18/ sociedad/338076007_850215.html

5. Dethlefsen, T. y Dahlke, R. (1998). La enfermedad como camino. Barcelona: Plaza \& Janés.

6. Feldman, R. (1998). Psicología. México: McGraw-Hill. 
7. Ferrater, J. (1971). Diccionario de fllosofía abreviado. Buenos Aires: Sudamericana.

8. Garrison, M. y Loredo, O. (2002). Psicología. México: McGraw-Hill.

9. González, P. (1981). Teorías sobre la creatividad (cap. 2). Recuperado de http://www.biopsychology.org/tesis_pilar/t_pilar04.htm

10. González López,H. (2012, 27 de abril). Teorías psicológicas y sus aportes a la creatividad. Recuperado de http://clubivanchukpollo. blogspot.com/2012/04/teorias-psicologicas-y-sus-aportes-la.html

11. González, M. (1992). I ching y psicologia transpersonal. Madrid: EDAF.

12. Meyer, P. (1996). La ilusión necesaria. Barcelona: Ariel.

13. Morris, C. y Maisto, A. (2001). Introducción a la psicología. México: Prentice-Hall.

14. Perls, F. (1987). Dentro y fuera del tarro de la basura. Santiago: Cuatro Vientos.

15. Schiffman, L. y Kanuk, L. (2001). Comportamiento del consumidor. México: Prentice Hall.

16. Sulloway, F. (1997). Rebeldes de nacimiento. Barcelona: Planeta.

17. Teicher, M. H. (2002, mayo de). Neurobiología del maltrato en la infancia. Investigación y Ciencia, 308, 59-67.

18. Wilhelm, R. (1982). I Ching. El libro de las mutaciones. Barcelona: Edhasa.

19. Yutang, L. (1953). La sabiduría de Lao-tsé. Buenos Aires: Sudamericana. 